\title{
Model petri net of adaptive traffic lights and its collaboration with a special event
}

\author{
Tomi Tristono ${ }^{1, *}$, Setiyo Daru Cahyono $^{2}$, Sutomo $^{3}$, and Pradityo Utomo ${ }^{1}$ \\ ${ }^{1}$ Informatics Department, Universitas Merdeka Madiun, 63133Madiun-East Jawa, Indonesia \\ ${ }^{2}$ Civil Engineering, Universitas Merdeka Madiun, 63133 Madiun-East Jawa, Indonesia \\ ${ }^{3}$ Mechanical Engineering, Universitas Merdeka Madiun, 63133 Madiun-East Jawa, Indonesia
}

\begin{abstract}
Traffic lights have an important role as the system control of vehicles flow on the urban network. Commonly, most countries still using fixed time strategy. Our research proposes the adaptive traffic lights model to response the traffic demand. It uses basic Petri net as a general modeling framework. Foractuating method of minimum and maximum green signal time interval, the green traffic lights have three-time extension units. Next, we collaborate on a case of the existence of railways that crosses on the southern arm of an intersection. We introduce both of collaboration model design of traffic lights and the railway's gate which always closes while a train passing. Verification and validation of the model are based on the simulation result of vehicles queue. The collaboration model design of traffic lights has excellent performance, and it can resolve the congestion problem better than conventional schedule.
\end{abstract}

\section{Introduction}

The primary role of the traffic lights is to improve the capacity and performance of junctions/ intersections in urban network area [1]. Traffic lights are installed to reduce congestion and travel delays, to make the main road crossable, to establish the time sharing fairly, to improve the safety of traffic vehicles and pedestrians, and so efficiency [2],[3].

Based on the time strategy, traffic lights are classified into two main categories, i.e., Fixed time strategy and traffic responsive strategy. The setting of the first strategy assumes that the traffic demand is constant. The second strategy is traffic lights that have the real-time control signal measurement [4]. It uses detectors to respond the fluctuation of traffic demand or special events [3].

The standard traffic lights have the sequence of green, yellow, and red signals that turn on in the specific time interval [5]. This paper's aim proposes modeling approach of adaptive traffic lights. This model is very beneficial to adjust to fluctuations in vehicles volume that passes on the road. Primarily in connection with the existence of a railway crossing. Of course, the number of railway's gate closures in the day will increase significantly during the beginning of double track of Railways era. It uses Petri Net (PN) to model traffic light behavior because they offer representation of conflict, sharing, synchronous and asynchronous, and priority [5]. Petri Net (PN) consisting of a four-tuple elements, i.e., places, transitions, arcs and tokens [6],[7].

The Marking places in a Petri Netcontaining a discrete number of tokens that the distribution represents a reachability of the net. An enabled transition of a Petri net may fire states while there are sufficient tokens in all of its input places. The firing of a transition consumes the required input tokens and deposits tokens in its output places [5].

A place may be refined into a subnet with many places and transitions resulting an expanded Petri Net [8]. That becomes the controller of the time interval of traffic lights.

\section{Traffic Lights Model}

This section reveals a green signal with a minimum interval time and maximum Petri Net model. It also explains the model of traffic lights that have two phases and P-invariants as the structural analysis to prove some properties of Petri Net.

A P-invariant indicates the tokens number in a set of places that satisfies some linear invariant.In a special case, all entries of a P-invariant are either 0 or 1 in which the number of tokens remains unchanged in all reachable markings [5].

\subsection{Petri Net and The Properties}

A Petri Net (PN) is a directed, weighted, and bipartite graph. The formal definition is written in the following: Petri net structure is the five-tuple elements $\mathrm{N}=(\mathrm{P}, \mathrm{T}$, Pre, Post, $\left.\mathrm{M}_{0}\right)$ [5],[6],[7].

$\mathrm{P}=\left\{\mathrm{p}_{1}, \mathrm{p}_{2}, \mathrm{p}_{3}, \ldots \mathrm{p}_{\mathrm{n}}\right\}$ is a places finite set, $\mathrm{P} \neq \varnothing, \mathrm{n} \in$ $\mathrm{N}+$. Places are ilustrated by circles. Places can represent signals, controlers, or events.

\footnotetext{
*Corresponding author: tomitristono@unmer-madiun.ac.id
} 
$\mathrm{T}=\left\{\mathrm{t}_{1}, \mathrm{t}_{2}, \mathrm{t}_{3}, \ldots \mathrm{t}_{\mathrm{m}}\right\}$ is a transitions finite set, $\mathrm{T} \neq \varnothing$, $\mathrm{m} \in \mathrm{N}+$. Transitions are illustrated by bars. The task of enabled transitions is as the trigger tools for signals, or events change. These are given the term fire.

Pre: $(\mathrm{P} \times \mathrm{T}) \rightarrow \mathrm{N}+$ is an input function that defines directed arcs from places to transitions.

Post: ( $\mathrm{T} \times \mathrm{P}) \rightarrow \mathrm{N}+$ is an output function that defines directed arcs from transitions to places. $\mathrm{N}+$ is the set of nonnegative integers. $\mathrm{M}_{0}$ is the initial marking.

Based on the reason that the model design must correct, the Petri Net (PN) desires some properties [5], [6], i.e., Reachability, Reversibility, Boundedness, Safeness [9], Liveness or deadlock free [5].A Marking M is to be reachable from the initial if there exists a sequence of transitions firing which transform a marking from the initial. The Marking must able to return to the initial marking, It is called as reversible. Boundedness is a control strategy to prevent overflow in the model. A place $p$ is said to be $k$-bounded if the number of tokens in $\mathrm{p}$ is always less than or equal to $\mathrm{k}(\mathrm{k} \in \mathrm{N}+)$ for every marking $\mathrm{M}$ that reachable from the initial marking. It is safe if it is 1-bounded.

\subsection{Green minimum and Maximum Time Interval}

To reduce the top-down Petri net model complexity, the traffic lights design must be performed in a modular way. It may be presented in parts of the system, the subsystems, and a part of the subsystems. The modularity can be specified and taken into account separately.

P_Green $[\alpha, \beta]$ at Fig. 1 (left) means that the minimum time interval of green light is $\alpha$ and the maximum is $\beta$. In this paper, the unit of time is second. A token exists in P_Green at least $\alpha$ unit of time, and It is not more than $\beta$ second. A token exists in $\mathrm{P}$ Green means that green signal turns on. It depends and adapts to the volume of traffic demand. P_Green_min $[\alpha, \alpha]$ means it has the minimum equal to the maximum time interval. P_Green_min $[\alpha, \alpha]$ has a fixed time interval, i.e.,the $\alpha$ unit of time. Each place P_Green_ext(1), P_Green_ext(2), and P_Green_ext(3) have time interval 4 seconds [5]. The Traffic Detector Handbook [10]gives a recommendation unit extension of 3.5 seconds for roads where speeds are higher than $30 \mathrm{~km} / \mathrm{h}$. For the simplicity, it is used 4 seconds.

Thistype of adaptive traffic light system has a simple algorithm.Manysystems implement complex algorithms and require new technologywhich is often an error. This minimum and maximum systems are easy to implement and durable [5].

\subsection{Two Phases Petri Net Traffic Lights Model}

A signalized intersection of the urban network consists of four arms. The traffic light setting has two phases i.e. SN (south-north) and EW (east-west) [11], [12]. The phase SN provides signals for traffic from the north and south arms simultaneously. The east arm traffic moves in one direction, entering the intersection and going to the south, west, and north arms. The traffic movement in the south and north arms have two directions, i.e., approaching and away the intersection. The traffic of vehicles come from the southern arm allowed to move towards the west and the north arm. The flows entering the intersection from the north arm can move towards the south arm only. Traffic flows in the western arm moves in one direction away from the intersection. Referring to government regulations, vehicular traffics is left-hand traffic (LHT).

The traffic light model can adapt to the traffic demand. The time interval specified from a minimum to a maximum value. A special situation existing at that intersection is the railways crossing on the south arm. The presence of the train on the railways arise as an obstacle to the traffic movement causing a congestion when a heavy traffic occurs. The railway's gate closure always longer than the time interval of a traffic lights cycle [11],[12].

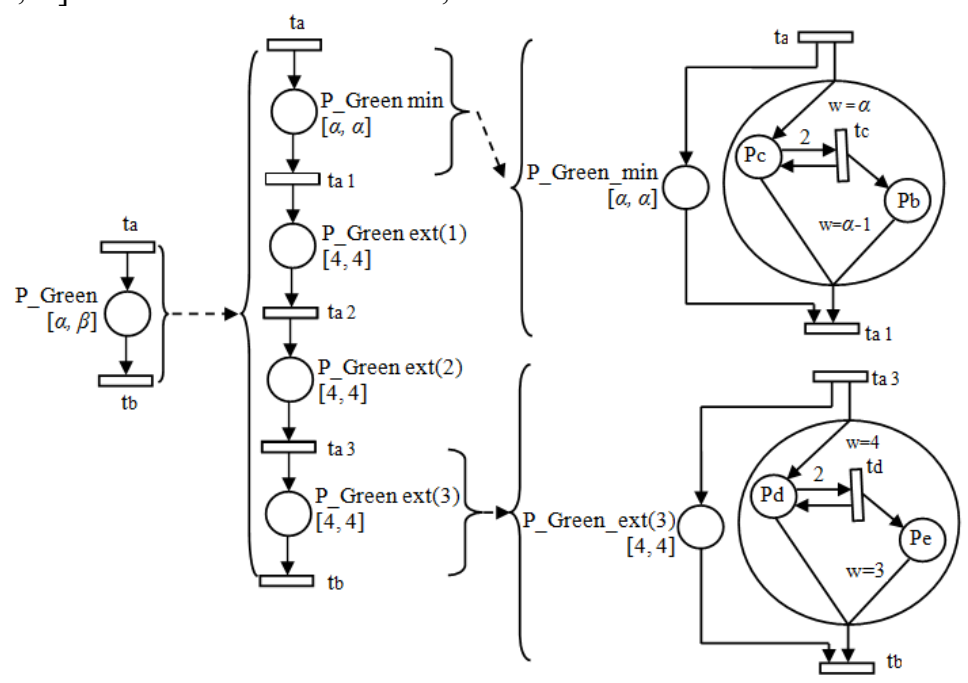

Fig. 1.At the left is the Place Green ( $\mathrm{P}$ Green is a signal model as Green signal). The refinement of $\mathrm{P}$ Green at the center and the big circle as Place controller (P controller) of the time interval at the right side. The controller is embedded in the P_Green. 


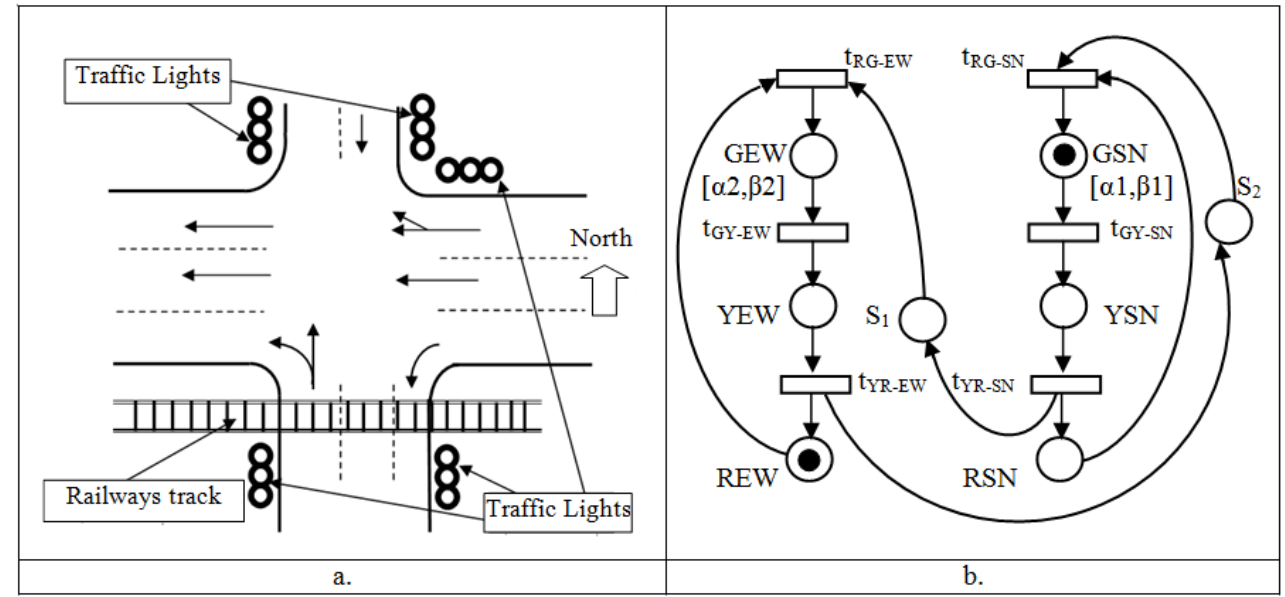

Fig. 2. a.The traffic flow of the intersection. b. The top-down Petri net model of a two-phases of traffic lights in normal schedule.

It refers to Fig. 2.b. Each GSN (Green South-North), and GEW (Green East-West) have refinement signal to adapt to the traffic demand. It defines signals YSN (Yellow South-North), RSN (Red South-North), YEW (Yellow East-West), and REW (Red East-West). Place $\mathrm{S}_{1}$, and $\mathrm{S}_{2}$ are the intermediation place to build the time order of Phase 1 (Phase South North) and Phase 2 (Phase EastWest). A cycle in each the traffic light phase represents the whole of the green, yellow, and red time interval [5].

\subsection{Signals Invariant of Two Phases Traffic Lights}

Signal invariants indicate set of signal places which they guarantee the number of tokens remains unchanged in all reachable markings. All entries of signal place invariants are either 0 or 1 . A signal turn on while a token exists in the signal place and the marking equal to 1 . It will be off when it is empty and the marking equal to 0 .

$$
\begin{aligned}
& M(G S N)+M(Y S N)+M(R S N)=1 \\
& M(G E W)+M(Y E W)+M(R E W)=1 \\
& M(G S N)+M(Y S N)+M(R S N)=M(R E W) \\
& \text { while } M(R E W)=1 \\
& M(G E W)+M(Y E W)+M(R E W)=M(R S N) \\
& \text { while } M(R S N)=1 \\
& M(G S N)+M(Y S N)+M(G E W)+M(Y E W)+M\left(S_{1}\right) \\
& +M\left(S_{2}\right)=1
\end{aligned}
$$

The invariant (1) presents that can be a token available in one of three signal places GSN, YSN, and RSN only of south-north traffic lights. It is similar for signal Places invariant (2).

The signal Places invariants (3) and (4) hint that the model provides the assurance of safety traffic movement. It refers to (3) while the red signal (REW) of east arm turns on, the traffic signals on the south and north arms are either GSN, YSN, or RSN. It is similar for (4) while red signals of the south and north arms go on.
The signal place invariant (5) uses intermediation places $S_{1}$ and $S_{2}$. These are the artificial signal places. While a token exists in places $\mathrm{S}_{1}$ or $\mathrm{S}_{2}$, the traffic lights turn on red signal for all phases.

\section{Method}

The method for actuating minimum and maximum green signal time interval applies three-time extension units. Each unit time interval is 4 seconds. The total extension is 12 seconds. The detectors inform to the controller for one unit of the first extension at least 2 seconds before the ending of the minimum green signal. The vehicular detectors collect the information for the second additional while first green extra going on, and it accumulates the instruction for the third additional while the second extension takes place. Sometimes while the low demand, the traffic lights do not require the green extension. The traffic light changes directly from Green minimum time interval to the yellow and redsignals.

The traffic lights collaborated to a case of the existence of railways that cross on the southern arm of an intersection. Weintroduce a collaboration model design of traffic lights. It employs two phases only. The model is the both collaboration of traffic lights and the railway's gate which always closes while a train is passing. Special events such as the closure of the railway's gate, entirely are always longer than the fulltime interval of a traffic light cycle.

The analysis and verification of traffic lights model use invariants, occurrence graphs, and Petri Net Simulator 2.0 for simulation [13]. At last, the performance test is also presented.

\section{A Case Study}

Acase is shown in Figure 2.a. The heavy vehicles (HV) dominate the flow entering the intersection of the eastern arm. Onthe contrary, the low vehicles (LV) and motorcycles (MC) are the largest parts of vehicles coming from the north and south arms. Bothconflicts of vehicle flow will be very dangerous.Updatingthe traffic flow schedule is very urgent and can not be delayed. 


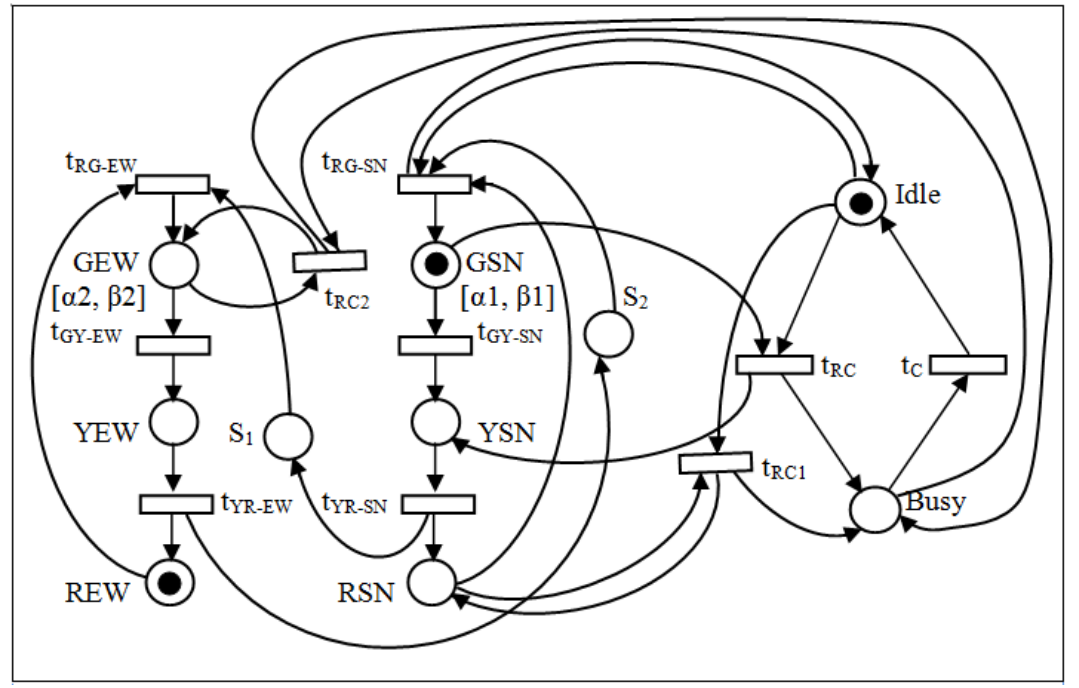

Fig. 3. The Petri net model of a collaboration of traffic lights and the railway's gate. It illustrated without control places of normal of traffic lights, the train ready to come, and completed passes.

\subsection{Design Model of The Railway's Gate and Its Collaboration}

Model of railway's gate in Fig. 3. has two places, namely Idle (when it open) or Busy (while closed). The place busy includes circumstances of the preparation for closing the railway's gate that must be longer than or equal to the time interval of YSN. Based on the reason of model simplification, it is not shown.

Transition $t_{\mathrm{RC}}$ (ready to come) and $t_{\mathrm{RC} 1}$ represent that the train ready to come and the railway's gate ready to close. The transition can be enabled if there are tokens in all its input signal places. The enabled transition can fire a token of the previous signal places to the next signal places. Transition $t_{R C}$ (ready to come) enabled if the train arrives while the Green South-North signal turns on. Otherwise, transition $t_{\mathrm{RC} 1}$ enabled if the railway's gate ready to close while red South-North signal turns on. It bases on the information of the train detectors. Transition $t_{C}$ (completed) informs that the train had crossed completely and the railway's gate ready to reopen.

The train is the priority. The signal model design in Fig. 3. has GSN minimum and maximum time interval. For the road users safety reason, It must have unlimited time interval connecting to the time interval of railway's gate closure. Sometimes while the train ready to come, it forces the green signal to turn yellow and then red, and it maintains the red signal until the train passes completely.

\subsection{Analysis and Verification of Petri Net Model Design}

While the train is passing and the railway's gate is closing, this means that the busy state is going on. Conversely, while the railway's latch is not closing, it means in the Idle state. In this state applies invariant (6), (7), and (8).

$$
M(B u s y)+M(\text { Idle })=1
$$

$$
\begin{aligned}
& M(B u s y)+M(G S N)=1 \\
& M(B u s y)+M(Y E W)+M(R E W)=1
\end{aligned}
$$

Places invariant (6) presents that the railway's gate state are either Busy or Idle. Places invariant (7) means that the south-north traffic lights may not turn on green signal while the railway's gate is busy. Places invariant (8) notes that while the railway's gate closes the eastwest traffic lights must turn on green signal only. Occurrence Graph (OG) shows that there is no possibility of a deadlock in the model. It is not presented here.

\section{Simulation Results and Discussion}

The Fig. 4. is the result of the verification and validation of the east arm vehicles flow. It uses "Passenger Car Equivalence" (PCE) to measure the traffic flow volume [14].

While the traffic flows is low, the frequency of minimum green time interval on the east arm is high. Very low traffic occurs in the midnightcausing traffic lights to always be lit with minimum interval time.

When medium traffic time interval in the early morning, the green time interval can be on additional time ext 1 , ext 2 , or maximum. The time ext 1 (28 seconds) is 21 times, time ext 2 ( 32 seconds) is 25 times, and the maximum green time interval (36 seconds) is five times.

On the peak hours, it can be ext 2 or the maximum green time interval. While the heavy traffic and a congestion after the railway's gate reopened again, the traffic lights are always lit at the maximum green interval time.

Basedon the reason the system algorithm is simple, the system is easily repaired when a little damage occurs. Thefour-second extension on the green time interval proved precisely to the actual demand of traffic on each arm. 


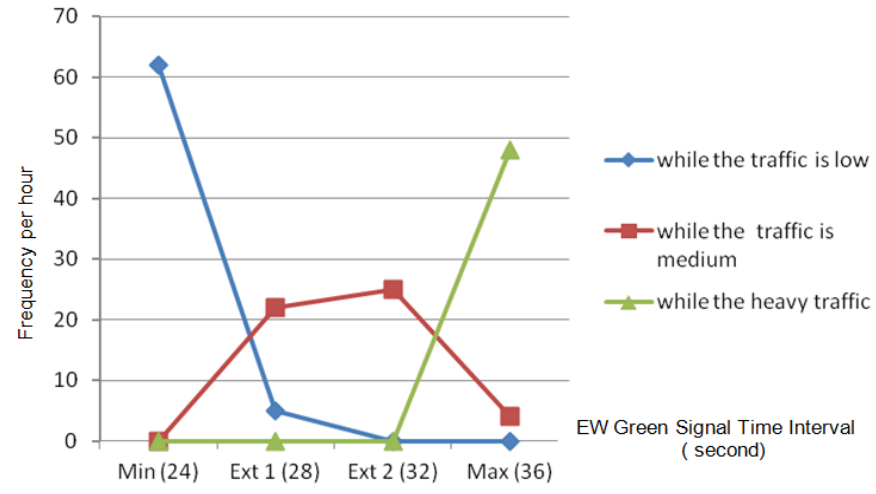

Fig. 4. The occurrencefrequency of each time interval of the green signal on three different traffic volumes of the east arm.

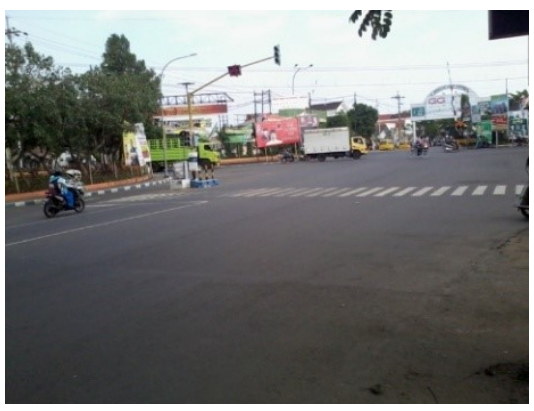

a.

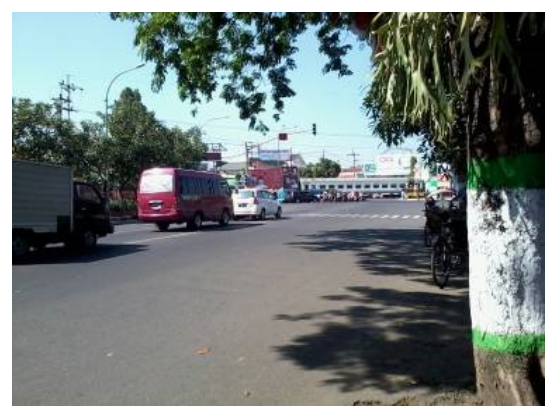

b.

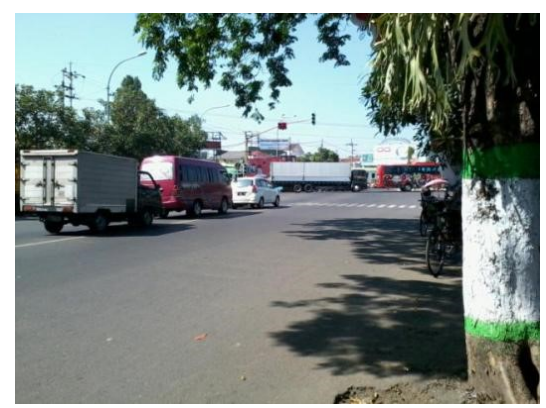

c.

Fig. 5.The real case that requires the way out of the problem. The location of the intersection is in the eastern of the Jombang City railway station. The photograph of intersection shooting is from the direction of the north arm. a. when a low vehicles traffic. b. When a low vehicles traffic and the train is passing in the southern arm. c. The heavy vehicles dominate the traffic flow from the east arm.

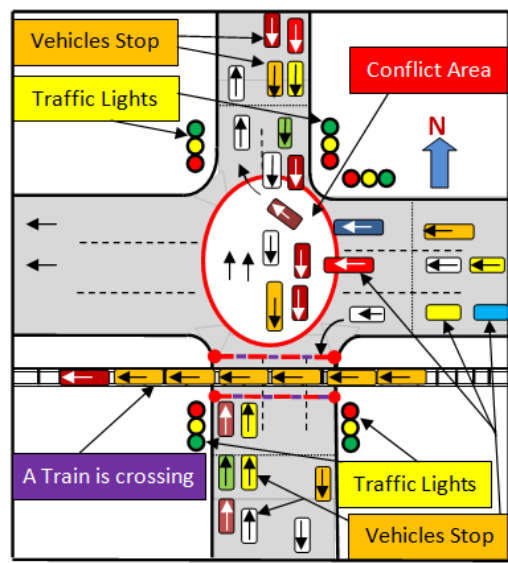

a.

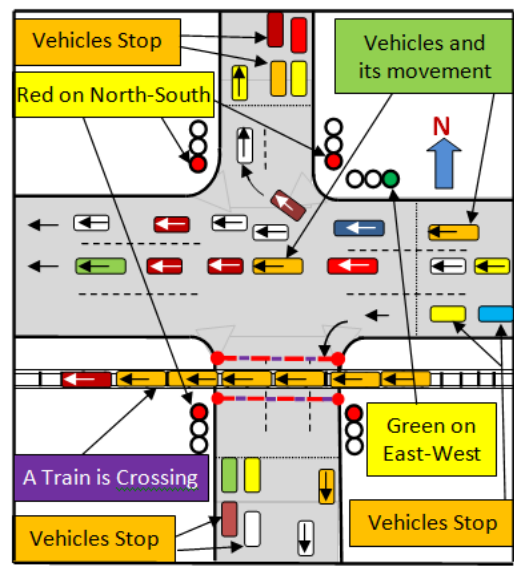

b.

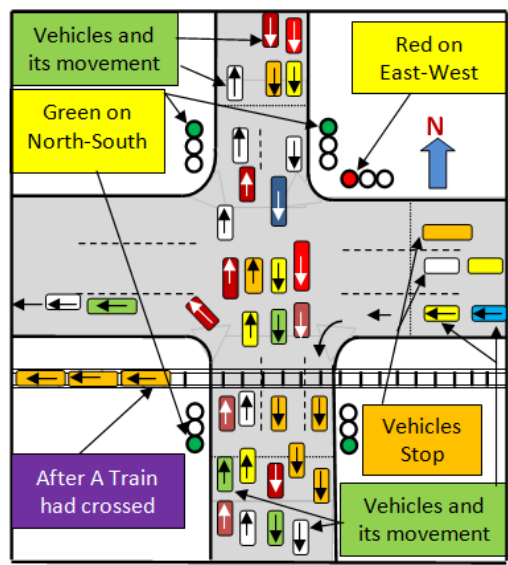

c.

Fig. 6.a. The visualization of the congestion while the railway's gate closure. The traffic lights schedule applied without collaboration. A chaotic situation occurs causing congestion in conflict areas of traffic flow. b. and c. present the vehicles flow settings that apply the collaboration traffic lights schedule to the railway's gate closure. b. while the train is crossing, EW green signal turns on, and c. after it completed, SN must turn on green signal at the first time.

Table 1. The schedules of two phases of traffic lights on the time interval of the maximum green signal

\begin{tabular}{|c|c|c|c|c|c|}
\hline \multirow{2}{*}{ Phases } & \multirow{2}{*}{ Green } & \multicolumn{2}{|c|}{ Inter Green } & \multirow{2}{*}{ Red } & \multirow{2}{*}{ Cycle } \\
\cline { 3 - 4 } & & Yellow & $\begin{array}{c}\text { All } \\
\text { red }\end{array}$ & & \\
\hline SN & 27 & 3 & 3 & 45 & 75 \\
\hline EW & 36 & 3 & 3 & 36 & 75 \\
\hline
\end{tabular}

There has been a railway's gate closure for 135 seconds during the peak hours. The time interval is longer than a cycle of traffic lights. The vehicles arriving has normal distribution on a measurement every 3 seconds. The vehicles leaving the intersection while the signal turned green has a negative exponential distribution. The simulation began when the traffic light started to turn red on the phase of the south-north/ SN.

In this paper, we present a graph of a queue of vehicles based on time. Thesecond form of presentation is the comparison of the number of vehicle queues of each arm by time-invariant.Inthe second form simultaneously displayed the pattern of each arm queue.

\footnotetext{
*Corresponding author: tomitristono@unmer-madiun.ac.id
} 


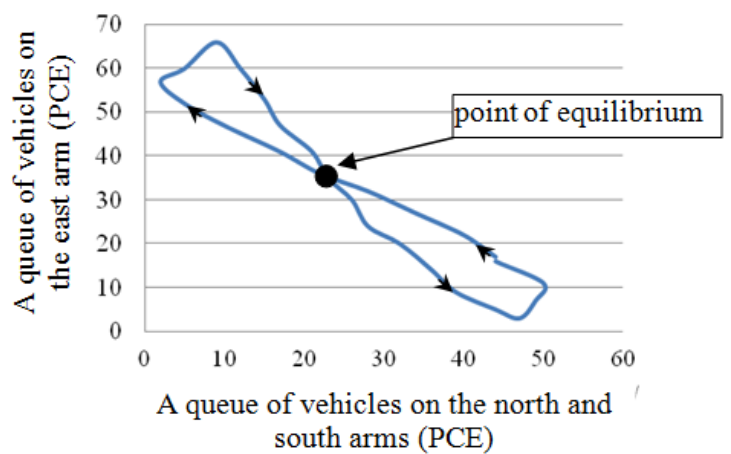

Fig.7. Vehicles queue in Normal Schedule (Presented using time-invariant)

Fig. 7 is the queue of vehicles due to the setting of the traffic lights. The vehicles queue goes up while the yellow or red lights are being lit and decreases when the green signal is on. A cycle of traffic lights is illustrated using time-invariant with an inclined lemniscate.

Fig. 8. presents the total sum of vehicles queue on the southern and the northern arm for 135 seconds of the railway's gate closure. The reopening of the railway's gate occurs at the ending of the north-south green signal. The setting traffic lights and the railway's gate are not collaborated. The congestion arising solved within six cycles. Each traffic lights cycle time interval is 75 seconds.

Fig. 9. describes a congestion in the southern and northern arm. The jamming on the east arm occurs also. A chaotic pattern illustrates the real situation at the intersection.

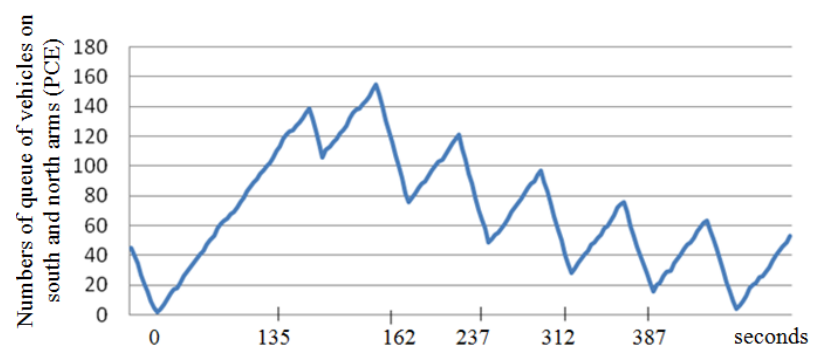

Fig. 8. The queue of vehicles of the south and north arms when the railway's gate closed and after it reopened. The settings without collaboration between light traffic and railway's gate. (Chart illustrates six cycles of traffic lights)

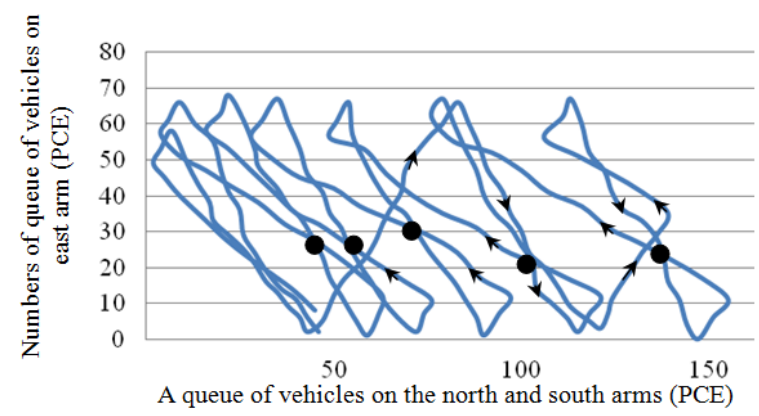

Fig. 9. The queue of vehicles of south-north arms and east arm when the railway's gate closed and after it reopened. The settings without collaboration between light traffic and railway's gate. (Chart illustrates six cycles of traffic lights). it uses time invariant.

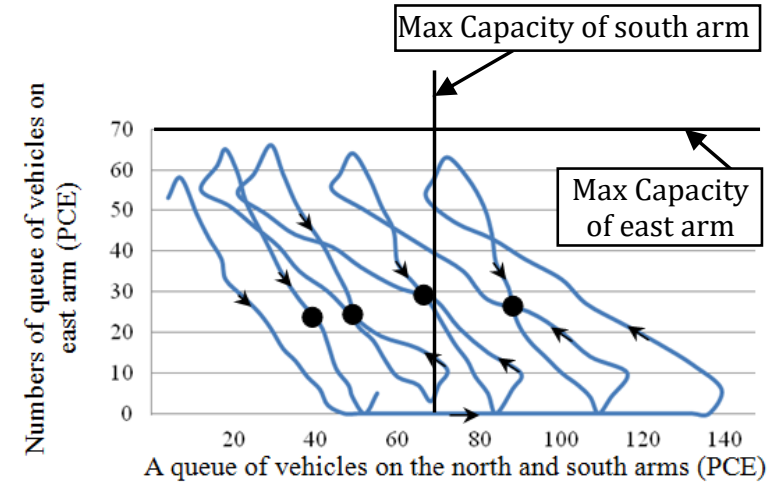

Fig. 10. Queue number of the vehicle when the train was passing and when the railway's gate has been reopened. Setting traffic lights implement collaboration with the railway's gate. (Illustrated four cycles of the traffic light after the railway's gate closure).

Fig. 10. shows the queue pattern implementing collaboration. At the beginning of the railway's gate closure, the queue of vehicles increase in accordance with the direction of the arrow. After the train has finished passing, the railway's gate reopened. The graph moves to left back. This means that the queue of vehicles decreased toward normal circumstances. It has a beautiful pattern. This illustrates the flow of vehicles in real situations that remain controlled. The bottlenecks are resolved in four cycles. It is two cycles faster when compared to the uncollaborated schedule.

The traffic lights setting applies collaboration able to reduce queues of vehicles coming from the east arm. The reopening of the railway's gate connected to the initial of the green signal for the north-south arm. The setting in this way is more efficient to ensure safety for all road users. The south-north traffic tends become impatient waiting after a long stop.

Fig. 10. is a visualization of the performance of the schedule of the collaboration of traffic lights to railway's gate. It looked better results to tackle congestion after an interruption. Whenthe railway's gate reopens, and peak hour, the traffic light applies the maximum green signal time.

The patterns while a low traffic flows has a similar shape, but smaller. The response of minimum and the maximum time interval of the traffic light are not visible, this due to traffic volume fluctuations that impossible occurred in a short period.

\section{Conclusion}

It has introduced an adaptive traffic lights model design with Petri net. This study also introduced a traffic light model that applies an interruption. This model is a collaboration between traffic lights and railway's gate closure. The simulation results show that this model has a better performance when compared to ordinary traffic light model to overcome the bottleneck after the closure of the railway's gate.

Acknowledgements. The research was funded by the Ministry of Research, Technology and Higher Education of Republic Indonesia on College Applied Research Scheme (PTUPT) in 
the year 2017. The contract number is No. 013/SP2H/HBPT/Unmer.Mdn/LPPM/ V/ 2017.

\section{References}

1. B. Yulianto, Setiono., FLATLTDBDR., KoNTekS 7, UNS,(2013).

2. M. Papageorgiou, C. Diakaki, V. Dinopoulou,A. Kotsialos, Y. Wang, RRTCS., Proc.of IEEE., 20432067, (2003).

3. Y.S. Huang, and T.H. Chung, MATLCSUTCPN, Intechopen.com, (2010).
4. F. Kurniawan, R.A. Al Hasibi,TCATCSDSMDWV, The Sci. J, UMY10,126-135, (2007).

5. M. Soares, ADIMLDSIS,Inf. F., Delft, 7, 99133,(2010).

6. C.G. Cassandras, S. Lafortune, DES, Kluwer Ac. Publisher, (1999).

7. D. Adzkiya, Subiono., MTLUPNIS,ITS-Sby, (2008).

8. C. Choppy, M. Mayero, L. Petrucci, EFPPNR, El. T. Comp. Sci. 214, 231- 254, (2008). 